\title{
High NA diffractive array illuminators and application in a multi-spot scanning microscope
}

\author{
B. Hulsken \\ D. Vossen \\ S. Stallinga \\ s.stallinga@tudelft.nl
}

Philips Digital Pathology Veenpluis 6, 5684 PC Best, The Netherlands

Philips Digital Pathology Veenpluis 6, 5684 PC Best, The Netherlands

Department of Imaging Science and Technology Delft University of Technology Lorentzweg 1, 2628 CJ Delft, The Netherlands

Array illuminators generating spots with high NA at high efficiency are presented. They are designed via application of high-NA scalar optics methods, and implemented as periodic binary phase structures. These array illuminators are used in a multi-spot scanning microscope for scanning large sample areas with a relatively high resolution.

[DOI: http://dx.doi.org/10.2971/jeos.2012.12026]

Keywords: diffractive optics, array illuminator, microscopy

\section{INTRODUCTION}

Spot array illuminators have been considered for optical interconnects [1, 2], microscopy [3], and data storage [4]. Optical designs in the sub-class of diffractive spot array illuminators are made utilizing the (fractional) Talbot-effect in some way or another [5]. All these cases deal with the Fresnel-regime of diffraction, which is suitable for low NA spots only (below approximately 0.25 ). Some cases propose a binary phase structure for generating the array of spots $[6,7]$, some even a hexagonal array [8], and some work in combination with an ordinary lens system [9].

Here we propose novel diffractive array illuminators which are suitable for generating spots with a Numerical Aperture (NA) up to 0.75 , thereby achieving higher compression ratio's (pitch to spot size ratio) then reported in the literature. This is achieved by abandoning the (fractional) Talbot-effect as guiding principle in the design of the diffractive structures and relying solely on numerical optimization, and expanding from the Fresnel-regime to high-NA (scalar) optics analysis. Furthermore, we report on an application of the array illuminator in a multi-spot scanning microscope, targeted at scanning large area tissue samples at a relatively high resolution, of the order of a $\mu \mathrm{m}$. Recently, a similar proposal has been put forward in ref. [10].

The paper is organized as follows. In Section 2 the design, manufacturing and test of the diffractive spot array generators are described, in Section 3 the implementation in a multi-spot scanning microscope is presented, Section 4 gives an overview of novel contrast modalities enabled by the technology, and Section 5 contains the conclusions.

\section{BINARY PHASE DIFFRACTIVE ARRAY ILLUMINATOR}

\subsection{Design}

The starting point of the design algorithm is the target spot array, which has an electric field within each unit cell equal to the Airy distribution:

$$
E_{\text {target }}(\vec{r})=\frac{2 J_{1}\left(k_{0} \mathrm{NA}|\vec{r}|\right)}{k_{0} \mathrm{NA}|\vec{r}|},
$$

with $k_{0}=2 \pi / \lambda$. This equation holds for a position $\vec{r}$ within one unit cell of size $p_{x} \times p_{y}$ and can be found within other unit cells by the assumed periodicity of the field. The 2D Fourier transform of the target field is:

$$
\begin{aligned}
\hat{E}_{\text {target }}(\vec{k}) & =\int_{U} d^{2} r E_{\text {target }}(\vec{r}) \exp (-i \vec{k} \cdot \vec{r}) \\
& =\frac{p_{x} p_{y}}{\pi} \operatorname{circ}\left(|\vec{k}| / k_{0} \mathrm{NA}\right) .
\end{aligned}
$$

In view of the periodicity of the spot array the Fourier transform of the target field has non-zero values only for $\vec{k}$ values on a discrete grid given by:

$$
\vec{k}_{j l}=\left(\frac{2 \pi j}{p_{x}}, \frac{2 \pi l}{p_{y}}\right) .
$$

Next we back propagate to the binary phase structure:

$$
\hat{E}_{\text {exit }}(\vec{k})=\exp \left(-i \Phi_{\text {prop }}(\vec{k})\right) \hat{E}_{\text {target }}(\vec{k})
$$

with the phase function for propagation:

$$
\Phi_{\text {prop }}(\vec{k})=d \sqrt{k_{0}^{2}-\vec{k}^{2}}+d_{\mathrm{cov}} \sqrt{n_{\mathrm{cov}}^{2} k_{0}^{2}-\vec{k}^{2}}
$$



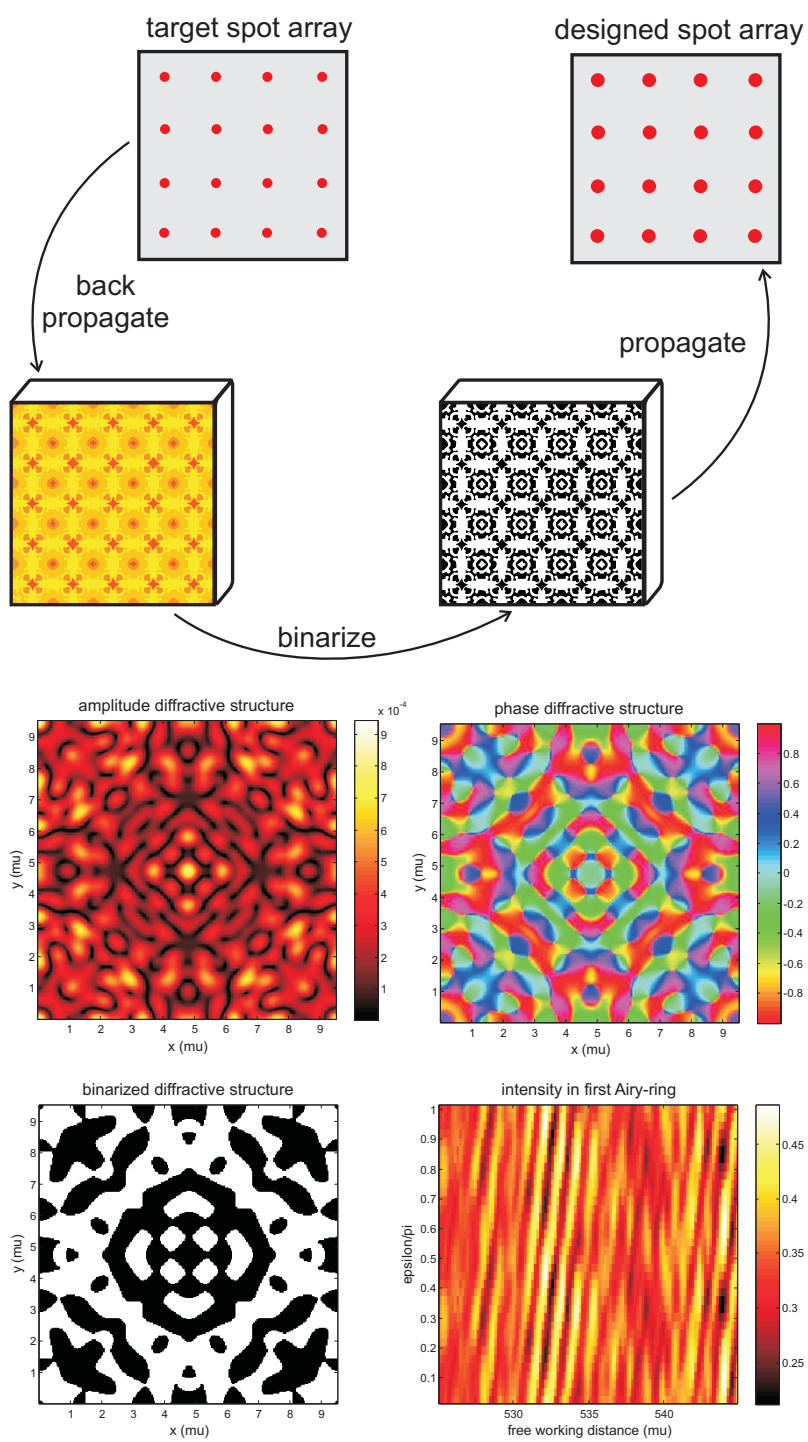

FIG. 1 Top: According to the design principle a target spot array consisting of Airy-spots with a target NA is back propagated to the exit surface of the spot generator plane, the resulting phase profile is binarized giving the actual design. Forward propagation to the spot array plane gives the actual spot shapes according to the design. Middle left: Amplitude of the target exit field of the spot array generator. Middle right: phase of the target exit field of the spot array generator. Bottom left: resulting binary phase structure. Bottom right, Power fraction within first Airy ring of the target pattern as a function of the free working distance and of the phase round-off parameter.

Here, $d$ is the free working distance, and $d_{\text {cov }}$ and $n_{\text {cov }}$ are the thickness and refractive index of the cover slip. The cover slip is taken into account because of the intended application in microscopy where the sample layer is sandwiched between a glass slide and cover slip. The diffractive structure then illuminates the sample through the cover slip. In our propagation phase factor we take effects of large propagation angles into account, but not effects of polarization, as we work in the scalar optics approximation. The next step is to take the inverse Fourier transform:

$$
E_{\text {exit }}(\vec{r})=\sum_{j l} \hat{E}_{\text {exit }}\left(\vec{k}_{j l}\right) \exp \left(i \vec{k}_{j l} \cdot \vec{r}\right)
$$

By construction, this field is periodic in both dimensions with periods $p_{x}$ and $p_{y}$. The phase of the binary phase structure follows as:

$$
\Phi(\vec{r})=\pi \bmod \left(\text { floor }\left\{\frac{\arg \left(E_{\text {exit }}(\vec{r})\right)+\epsilon}{\pi}+\frac{3}{2}\right\}, 2\right),
$$

where $\varepsilon$ is the phase round-off parameter. The transmission function is:

$$
T(\vec{r})=\exp (i \Phi(\vec{r})) .
$$

The design principle is schematically summarized in Figure 1. It is stressed that although there is one spot per unit cell, there is no one-to-one correspondence between unit-cells and spots. In view of the large NA and large free working distance each spot receives complex amplitude contributions from many unit-cells, and vice versa each unit cell contributes complex amplitude to a multitude of spots. This stands in contrast to the use of spot arrays generated by micro-lens arrays for which there is a one-to-one correspondence between each unit-cell/micro-lens and each spot of the spot array. Figure 1 also shows plots of the amplitude, phase and resulting binary phase structure for a typical design, with design parameters $\lambda=655 \mathrm{~nm}, \mathrm{NA}=0.75, p_{x}=p_{y}=9.504 \mu \mathrm{m}, d_{\mathrm{cov}}=145 \mu \mathrm{m}$, $n_{\text {cov }}=1.5142, d=532 \mu \mathrm{m}, \varepsilon=0.39 \pi$, and for a unit cell (and Fourier spectrum) discretized on a $256 \times 256$ lattice. The typical feature size of the resulting binary phase structure is determined by the target NA, i.e. by the largest wavevector with substantial contribution to the spot. The feature size is typically of the order $\lambda / 8 \mathrm{NA}$, which is about $100 \mathrm{~nm}$. The amplitude and phase are highly irregular, with a number of phase singularities (and attending amplitude zeros) visible. At first sight, the approximation of the required exit field with a binary phase structure seems to be a too coarse approximation to result in reasonable quality spot arrays. However, it appears that, fortuitously perhaps, the results are not that bad at all, and quite reasonable spots can be produced.

A huge variety of esthetically pleasing structures can be generated by varying the free parameters of the design. Figure 2 shows movies of the found binary phase structures for variations in the phase round-off $\varepsilon$ and for variations in the free working distance $d$. Starting point is the design of Figure 1, and that particular binary phase structure can be found as one of the frames of the movies. Intriguingly intricate structures are also revealed by making a through-focus scan of the light distribution within a unti-cell. A movie of such a throughfocus scan is also shown in Figure 2 for the design of Figure 1.

In order to assess the spot quality for the designed structure the design procedure is reversed. First we take the Fourier transform of the binary phase transmission function:

$$
\hat{T}(\vec{k})=\int_{U} d^{2} r T(\vec{r}) \exp (-i \vec{k} \cdot \vec{r}),
$$

then we restrict the angular spectrum to propagating waves and multiply with the propagation phase factor:

$$
\begin{aligned}
\hat{E}_{\mathrm{im}}(\vec{k})= & \exp \left(i \Phi_{\text {prop }}(\vec{k})\right) \operatorname{circ}\left(|\vec{k}| / k_{0}\right) \hat{T}(\vec{k}) \\
& \times \exp (-i \vec{k} \cdot \vec{r})
\end{aligned}
$$

and we find the actually imaged spot array field by applying yet another Fourier transform as:

$$
E_{\mathrm{im}}(\vec{r})=\sum_{j l} \hat{E}_{\mathrm{im}}\left(\vec{k}_{j l}\right) \exp \left(i \vec{k}_{j l} \cdot \vec{r}\right) .
$$


phase round - off $=-0.48611$

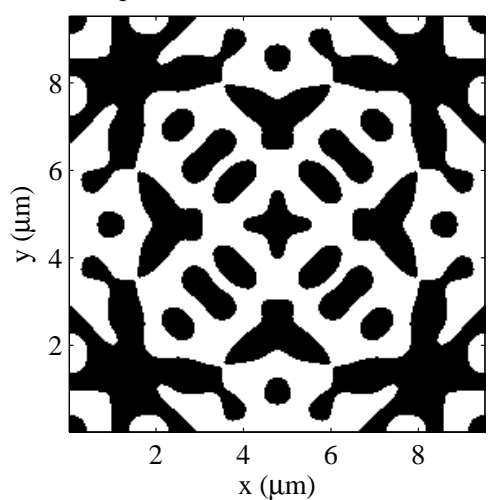

free working distance $=530 \mathrm{mu}$

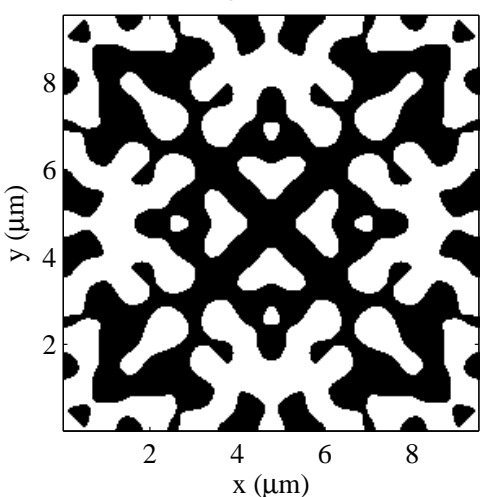

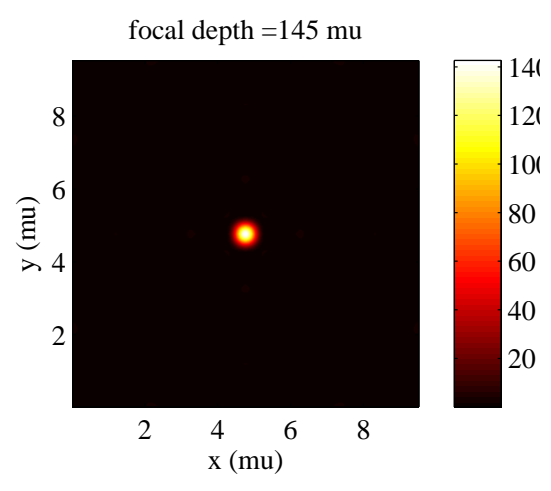

FIG. 2 Left: Movie showing the effect of the phase round-off parameter $\varepsilon$ on the designed periodic binary phase structure (size: $863 \mathrm{kB}$, format: avi, file: Figza.avi). Middle: Movie showing the effect of the free working distance $d$ on the designed periodic binary phase structure (size: 949 kB, format: avi, file: Fig2b.avi). Right: Movie showing the effect of defocus on the free working distance $d$ on the designed periodic binary phase structure (size: 1557 kB, format: avi, file: Fig2c.avi).
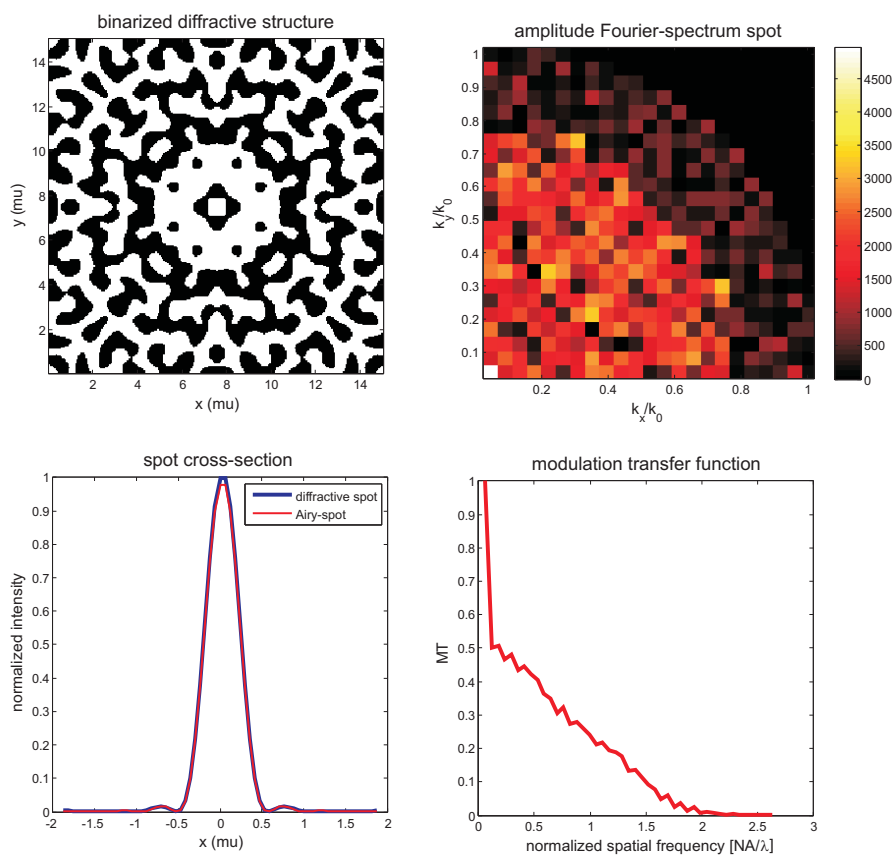

FIG. 3 Example of a local optimum design. Top left: The binary phase structure, top right: The amplitude of the Fourier spectrum giving rise to the spot array, bottom left: Spot cross-section and for reference the target Airy-spot cross-section, bottom right: Modulation transfer function calculated from the FT of the spot intensity.

The measured intensity is proportional to:

$$
I_{\mathrm{im}}(\vec{r})=\left|E_{\mathrm{im}}(\vec{r})\right|^{2}
$$

and a measure for the spot quality is the summed intensity within the first Airy ring of the target spot at $R=0.61 \lambda / \mathrm{NA}$

$$
Q=\frac{1}{P} \int_{|\vec{r}| \leq R} d^{2} r I_{\mathrm{im}}(\vec{r}),
$$

with $P$ the power incident on one unit-cell, and depends only on the two design degrees of freedom, namely the free working distance $d$ and the phase round-off $\varepsilon$. Figure 1 shows a plot of a typcal design and of $Q$ (normalized to the incident power on each unit cell) as a function of these parameters (all other parameters kept the same as before). The merit function landscape is quite irregular with many local maxima, all extending to a maximum of about 0.5 . This is about $60 \%$ of the value of

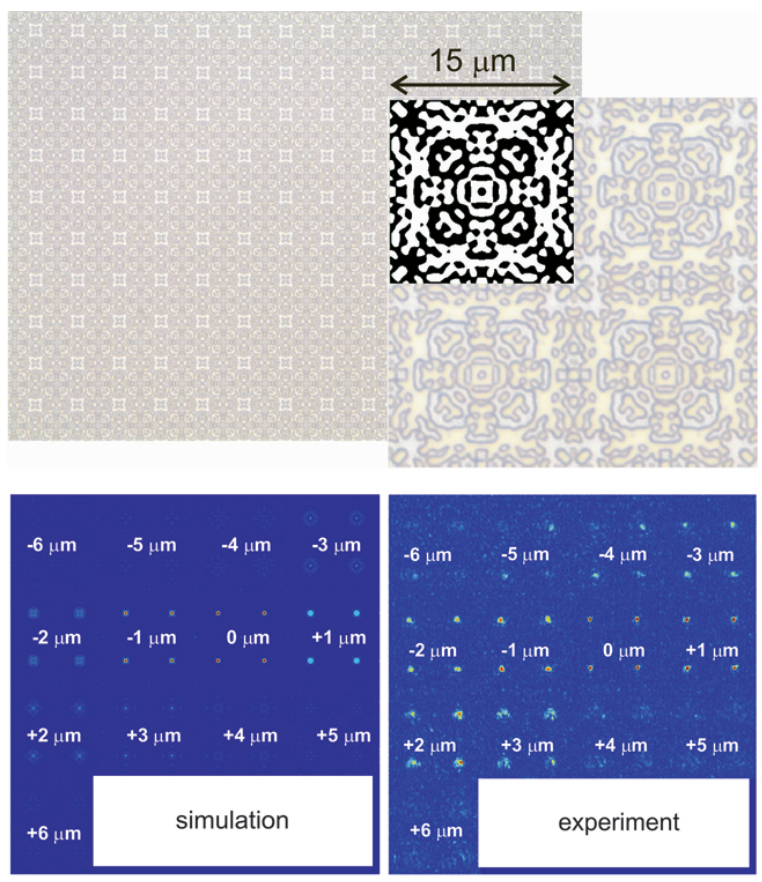

FIG. 4 Top: Microscopic image of realized binary phase structures for generating spot arrays, and the original design superimposed on the inset. Bottom: Through focus images of a subset of four spots according to simulation (left) and experiment (right).

0.84 obtained in case the imaged spot array would match exactly with the target spot array. These saliant features of the merit function landscape have been observed for all regions in the design space considered but we have no explanation of the underlying mechanism. Finally, it is mentioned that the design philiosophy presented here has previously been described in ref. [11].

One such local optimum (for $\lambda=658 \mathrm{~nm}, \mathrm{NA}=0.75$, $p_{x}=p_{y}=15 \mu \mathrm{m}, d_{\mathrm{cov}}=145 \mu \mathrm{m}, n_{\mathrm{cov}}=1.5142$, $d=577.5 \mu \mathrm{m}, \varepsilon=0.39 \pi$ ) is shown in Figure 3 . This design reaches a quality measure $Q=0.45$, the resulting spot resembles quite closely the targeted Airy-spot, the background level (about half the power for a quality measure $Q=0.45$, distributed over the entire unit-cell area) is not visible in this graph. The MTF nicely follows the expected near-linear decrease to the cut-off at $2 \mathrm{NA} / \lambda$, with a DC-peak 


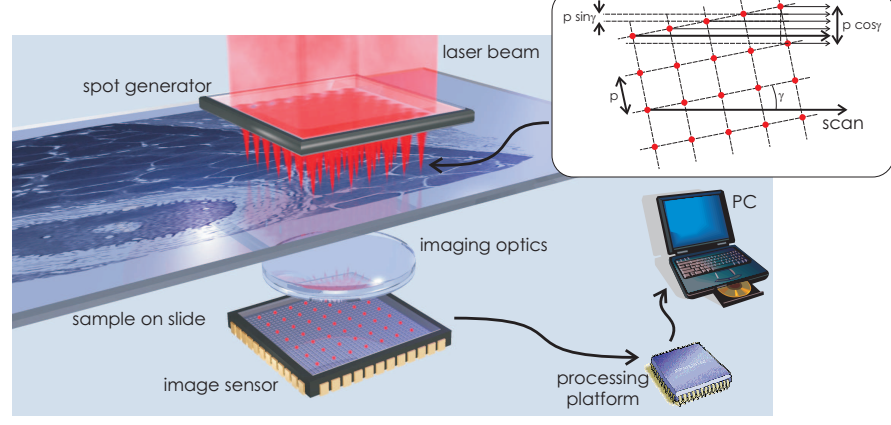

FIG. 5 Principle of the multispot scanning microscope (courtesy Jean Schleipen, Philips Research).

of about $50 \%$ height. This fits with the value of $Q=0.45$, as that implies that a little more than half the incident power is not focused into the spot, i.e. contributes to a (near) uniform background. A uniform background gives rise to a peak in the MTF at zero spatial frequency.

\subsection{Test}

Periodic binary phase structures for generating spot arrays have been made by direct e-beam writing in resist and subsequent etching in silica $(n=1.51)$ to a depth $h=\lambda / 2 /(n-1)$. The e-beam diameter of about $25 \mathrm{~nm}$ enables the subwavelength features needed to produce spots with an NA close to one. It is envisioned that these spot generators can be mass-manufactured at low cost by embossing a master mold into a suitable polymer, similar to the production of optical discs, which have comparable feature sizes and depth. Figure 4 shows actually realized binary phase structures for a design with a pitch $p=15 \mu \mathrm{m}$, a red diode laser wavelength $\lambda=650 \mathrm{~nm}$, and a free working distance of $458 \mu \mathrm{m}$, and for focusing through $140 \mu \mathrm{m}$ glass with refractive index $n=1.52$. The figure also shows through-focus images of a subset of four spots of the array. The experimental FWHM of typical spots was $0.49 \mu \mathrm{m}$, compared to $0.45 \mu \mathrm{m}$ for the design, making the effective NA about equal to 0.69, compared to the design value of 0.75 . In addition, the background level appeared to be higher than in the simulations. These differences may be attributed to a difference between the design wavelength and the actual wavelength $(\lambda=658 \mathrm{~nm})$ in the experiment, and also to speckle effects.

\section{MULTI-SPOT SCANNING MICROSCOPE}

Figure 5 schematically shows a multi-spot scanning microscope based on the diffractive spot array generator. The array of spots is used to illuminate a sample. The sample is placed on a translation stage and scanned at constant velocity $v$ in a direction that makes a small angle $\gamma$ with the $L_{x} \times L_{y}$ spot array. As a consequence the $L=L_{x} L_{y}$ spots scan $L$ equidistant scan lines. The distance between the scan lines is $R / 2$ with $R$ the sampling distance. The transmitted light is imaged by an optical relay system onto a pixelated image sensor which captures images at a framerate $f=R / 2 v$. These image are analyzed by a fast processing platform for identifying the spot grid positions and finding the transmittance for each spot of the array. This results in the final image that can be stored on a hard-drive and displayed on a computer screen. The resolution is given by the wavelength, by the array illumination NA and by the collection NA:

$$
R_{\mathrm{opt}}=\frac{\lambda}{\mathrm{NA}_{\mathrm{ill}}+\mathrm{NA}_{\mathrm{col}}}
$$

In case of Nyquist-sampling the sampling distance is equal to the resolution $\left(R=R_{\text {opt }}\right)$, in case of oversampling it is smaller ( $R<R_{\text {opt }}$ ), in case of oversampling it is larger $\left(R>R_{\text {opt }}\right)$. A relatively high resolution is guaranteed by the relatively high value of the array illumination NA, which can easily be as high as $\mathrm{NA}_{\mathrm{ill}}=0.75$ for an air-incident configuration, and even higher for an immersion-type of setup. It follows that the collection NA can be considerably smaller (provided enough light is captured to achieve sufficiently high signals on the image sensor), which can be used by aiming at imaging systems with a relatively large field of view (e.g. microscope objectives tend to have larger fields of view at lower NA). Another key advantage of the proposed multi-spot scanning microscope is that it entirely relies on components with low-to-moderate cost, enabling a novel balance between a relatively high resolution, a large field of view and cost-effectiveness.

The onedimensional scanning strategy requires that the array size in the $x$-direction $L_{x}$, the angle $\gamma$, the resolution $R$ and the pitch $p$ are related by:

$$
\begin{aligned}
& p \sin \gamma=R / 2, \\
& p \cos \gamma=L_{x} R / 2 .
\end{aligned}
$$

Finally, it is mentioned that, interestingly, this way of onedimensional scanning of a large field with an array of spots by rotating the array has been invented at least six times [12]-[17].

The image processing needed to distill the spot grid comprises the steps of (1) thresholding each recorded image using Otsu's method, (2) labeling all distinct regions above threshold, (3) noise reduction by median filtering on these regions, (4) finding the centroid positions to sub-pixel accuracy for all labeled regions, (5) creating the Delaunay triangulation to the set of centroid positions, (6) selecting the triangles with near $\pi / 2$ corner angle, (7) finding the pitch in $x$ and $y$ from averaging the sides of the triangles in this sub-set, (8) fitting the offset of a rectangular grid with the the found rectangular unit cell to the spot positions. For each unit cell surrounding each grid position the maximum tranmission value (spot peak), the centroid position of the spot, and the integrated transmission value over a specified region of interest smaller than the unit cell (electronically defined 'pinhole') can be calculated. The nominal centroid positions, averaged over many frames, is indicative for the optical distortion of the optical system that images the sample to the sensor, which can thus be corrected for. The grid fitting procedure results in time traces of the measured quantity for each of the spots of the scanning spot grid. These time traces can be ordered to represent consecutive scan lines according to the position in the $L_{x} \times L_{y}$ grid. Finally, the relative delay between the scan lines can be removed using the value for the rotation angle $\gamma$ and using the known relation between the scan line and the spot position of the in the $L_{x} \times L_{y}$ grid. 

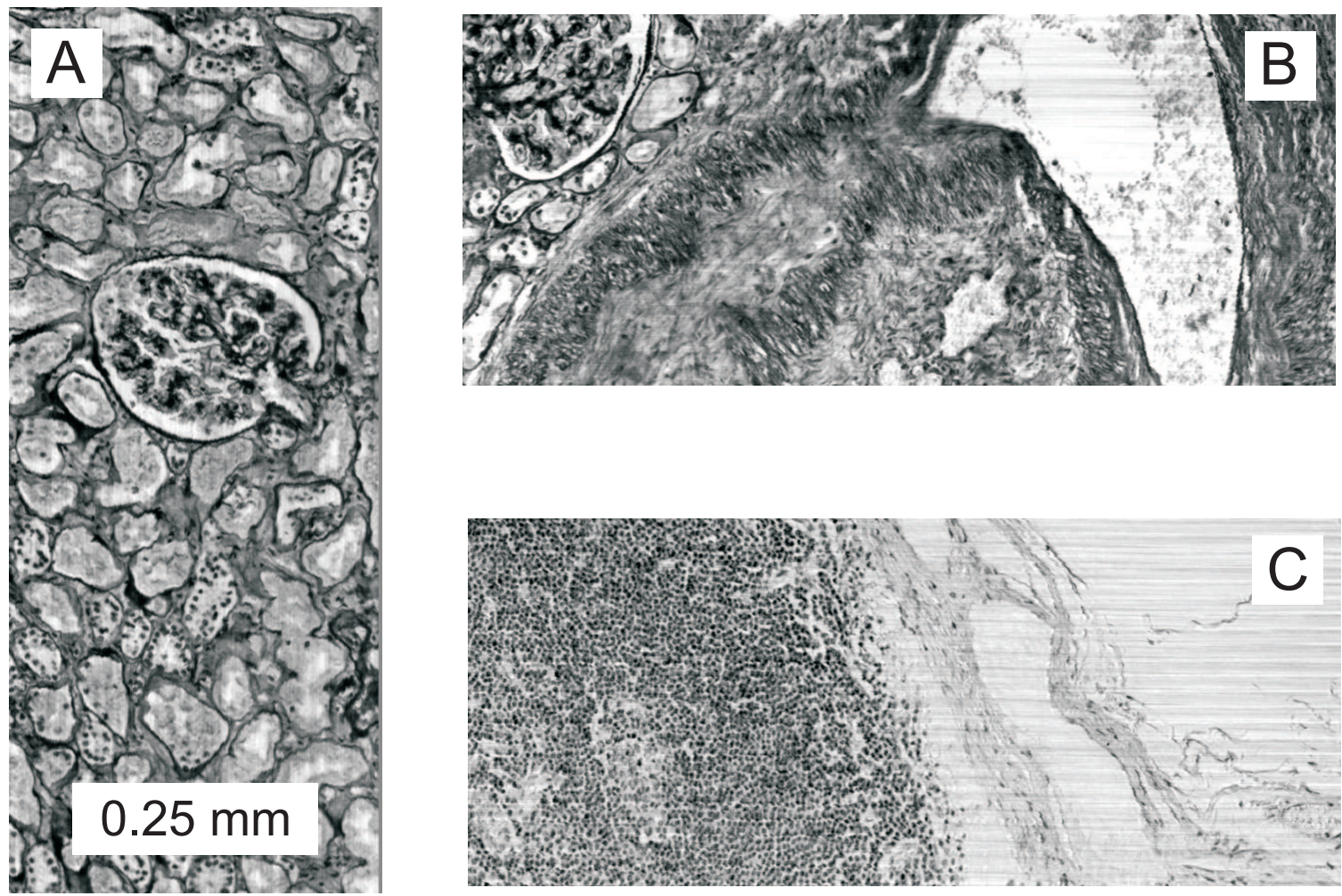

FIG. 6 Images recorded with the multi-spot scanning microscope of kidney tissue (A and B) and of lymph gland tissue (C). The tissue samples are standard hematoxylin $れ$ eosin stained to give an absorption contrast.

Figure 6 shows examples of tissue slides scanned with an experimental setup built according to the principles outlined above. The setup used a red laser diode and spot generator as described above, an optical system for imaging the sample onto the image sensor consisting of an N Plan $20 \times / 0.40$ Leica objective lens (focal length $200 \mathrm{~mm} / 20 \times=10 \mathrm{~mm}$ ) with spherical aberration correction ring for imaging through a $1 \mathrm{~mm} \mathrm{mi-}$ croscope slide, and a Melles-Griot $F=125 \mathrm{~mm}$ tube lens, giving a total magnification equal to 12.5 . The image sensor was a Micron MT9V403 VGA $(640 \times 480$ pixels) sensor with framerate up to $200 \mathrm{fps}$ and with $9.9 \mu \mathrm{m}$ pixels, giving a unit-cell on the sensor of about $19 \times 19$ pixels, and a total of $32 \times 25$ unitcells, comprising a total of 800 spots, that are used for imaging. The rotation angle follows as $\gamma=\arctan (1 / 32)=1.8 \mathrm{deg}$, and the sampling distance as $p \sin \gamma=0.47 \mu \mathrm{m}$. In view of the optical resolution limit $R=658 \mathrm{~nm} /(0.69+0.40)=0.6 \mu \mathrm{m}$ this means that we are somewhat undersampling. The image analysis was done on a PC using Matlab. Possible follow-up systems may profit from high-speed parallel processing platforms such as graphics cards. The image quality appears to be primarily limited by array non-uniformity, probably induced by speckle. This is partly compensated by a calibration step for normalizing the nominal intensity of each scan line to an average value. Nevertheless, a residual 'blinding' artefact is clearly recognizable. This artefact highlights the boundaries between the different scan zones (each zone consists of the lines generated by one row of the spot array).

\section{NOVEL CONTRAST MODALITIES}

A drawback of the proposed multi-spot scanning microscope is that it is inherently monochratic. Acquiring colour images would entail switching laser-spot generator combinations, but even then only a discrete set of wavelengths would make up the image, not a broad continuous band of wavelengths. However, the combination of diffractive spot engineering along the lines of the presented design method and analyzing the images acquired with the sensor makes several novel contrast modalities possible. Here, two possibilities are discussed.

\subsection{Multifocal spot array}

Instead of one spot per unit cell a plurality of $M$ spots per unit cell can be engineered. For example, each of the $M$ spots can have a focus at a different depth, thereby allowing to scan $M$ focal slices simultaneously. These $M$ focal slices can also be used for auto-focus operation or for multi-focal phase contrast [18].

Figure 7 shows an actual design of a binary phase structure for generating three arrays of spots for focusing at three different depths $\left(\lambda=655 \mathrm{~nm}, \mathrm{NA}=0.65, p_{x}=2 p, p_{y}=p\right.$, $p=9.504 \mu \mathrm{m}, d_{\mathrm{cov}}=145 \mu \mathrm{m}, n_{\mathrm{cov}}=1.5152, d=518.2 \mu \mathrm{m}$, $\varepsilon=0.33 \pi$ ), separated by $\Delta z=2.5 \mu \mathrm{m}$. Cross-section through the spots show that indeed three distinct spots are formed at the focal depth values given by the design parameters.

\subsection{Spot displacement phase gradient contrast}

The spot measured at the sensor has a different height and centroid position compared to the nominal grid position. Restricting the signal values to the pixels close to the grid position effectively defines a pinhole, thus emulating confocal imaging. This would have the advantage of improving con- 

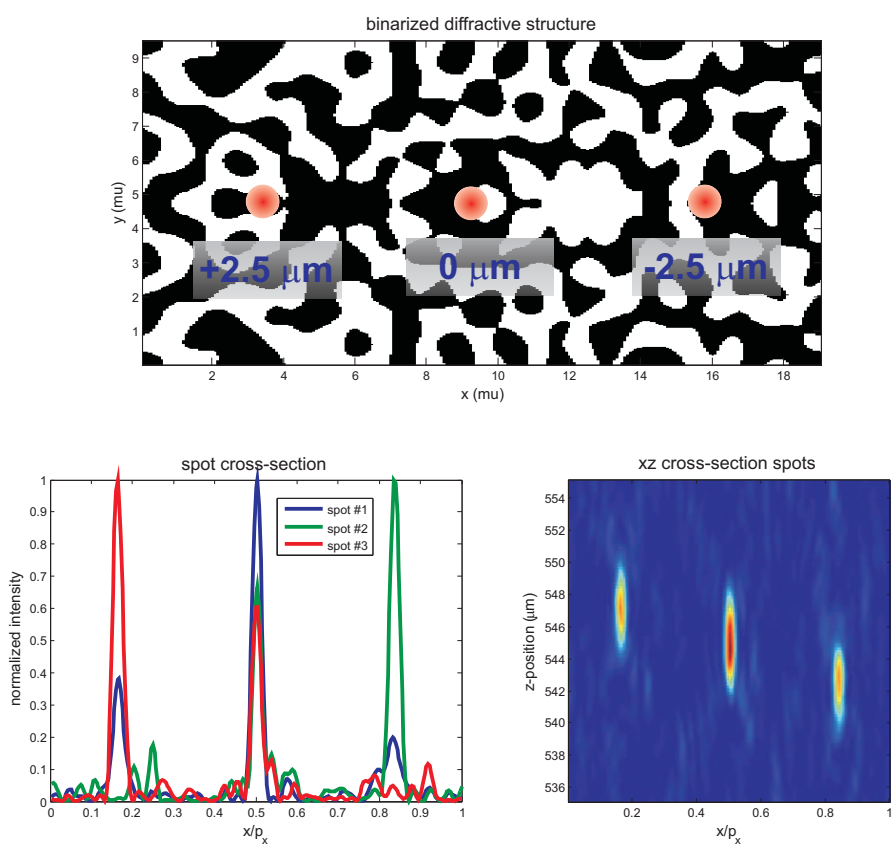

FIC. 7 Top: Binary phase structure for generating a multifocal spot array, the red dots indicate the lateral position of the three spots within the $2 p \times p$ rectangular unit cell, the axial separation is $2.5 \mu \mathrm{m}$. Bottom left: Cross-section along the horizontal heart line through the three spots for the three depths at which the three spots come into focus, bottom right: Cross-section of the spots in the $x z$-plane.

trast in imaging thick samples, as the light scattered by out-offocus layers is filtered out. The displacement of the spot from the nominal grid position is an entirely new contrast modality, and gives rise to images similar to Differential Interference Contrast (DIC) [19]. In particular, this modality is sensitive to the (gradient of the) refractive index.

In order to understand how this can arise consider a thin phase grating with small modulation depth $\Delta \phi$ and relatively large pitch $p$ placed at a defocus $\Delta z$ from the object plane. The transmission function of the grating is then:

$$
\begin{aligned}
T(x) & =\exp (i \Delta \phi \cos (2 \pi x / p)) \\
& \approx 1+\frac{1}{2} i \Delta \phi \exp (2 \pi i x / p)+\frac{1}{2} i \Delta \phi \exp (-2 \pi i x / p) .
\end{aligned}
$$

The electric field after the grating then follows as the sum of the 0 th, +1 st and -1 st diffraction orders, where the \pm 1 st orders are displaced over a distance $\lambda \Delta z / p$ :

$$
\begin{aligned}
E(x)= & E_{0}(x)+\frac{1}{2} i \Delta \phi E_{0}(x-\lambda \Delta z / p) \\
& +\frac{1}{2} i \Delta \phi E_{0}(x+\lambda \Delta z / p),
\end{aligned}
$$

with $E_{0}(x)$ the electric field of the illumination spot. The resulting intensity follows as:

$$
\begin{aligned}
I(x)= & |E(x)|^{2} \approx\left|E_{0}(x)\right|^{2}-\frac{2 \lambda \Delta z \Delta \phi}{p} \\
& \times \sin (2 \pi x / p) E_{0}(x) \frac{d E_{0}(x)}{d x}
\end{aligned}
$$

where it has been used that the displacement of the diffraction orders is assumed to be small compared to the spot size. We then find that:

$$
I(x) \approx I_{0}\left(x-\frac{\lambda \Delta z \Delta \phi}{p} \sin (2 \pi x / p)\right),
$$
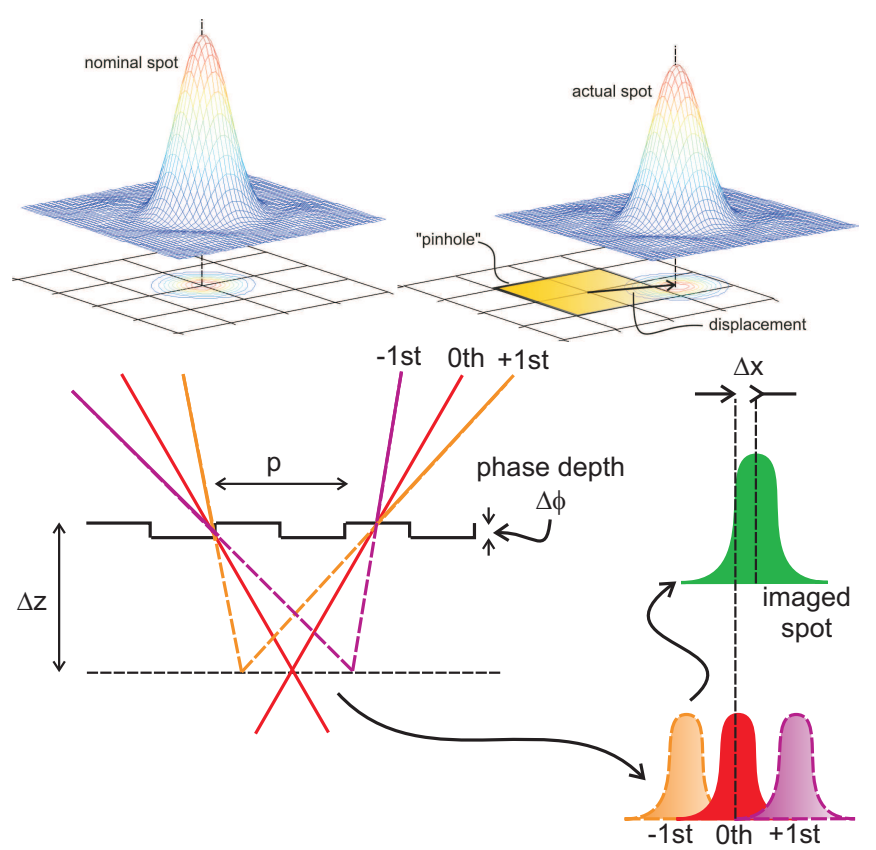

FIG. 8 Top: The principle of confocal and spot displacement phase contrast imaging with the multi-spot scanning microscope. Bottom: relation spot displacement and phase gradient contrast.

and a spot displacement equal to $-(2 \pi \Delta z \Delta \phi \lambda / p) \sin (2 \pi x / p)$. This may be generalized to any 2D slowly varying thin phase object $\Phi(\vec{r})$ which gives rise to a spot displacement:

$$
\delta \vec{r}=-\frac{\lambda \Delta z}{2 \pi} \vec{\nabla} \Phi
$$

Clearly, the spot displacement is proportional to the phase gradient, implying that this is a DIC-like contrast modality. The accuracy of the phase gradient measurement follows from the accuracy of measuring the spot displacement. This is typically equal to $\lambda / \mathrm{NA} / \sqrt{N}$, with $N$ the number of collected photons. It follows that the accuracy of measuring the phase gradient is typically equal to $2 \pi /(\Delta z \mathrm{NA} \sqrt{N})$. Consequently, for a sufficiently high photon count the accuracy can be quite advantageous.

\section{CONCLUSION}

In summary, we have presented a simple and straightforward method for designing high NA diffractive array illuminators. These have been realized by e-beam lithography, the measured spot quality is very good and close to expectations based on the design. The array illuminators have been applied in a multi-spot scanning microscope for scanning a large area with a relatively high resolution. The combination of engineering the spot properties by diffractive design and intricate processing of the raw sensor images provides novel contrast modalities, in particular multifocal imaging and spot displacement phase gradient contrast.

\section{References}

[1] A. W. Lohmann, "An array illuminator based on the Talbot effect," Optik 79, 41-45 (1988). 
[2] A. W. Lohmann, and J. A. Thomas, "Making an array illuminator based on the Talbot effect," Appl. Optics 29, 4337-4340 (1990).

[3] T. Tanaami, S. Otsuki, N. Tomosada, Y. Kosugi, M. Shimizu, and H. Ishida, "High-Speed 1-Frame/ms Scanning Confocal Microscope with a Microlens and Nipkow Disks," Appl. Optics 41, 4704-4708 (2002).

[4] R. F. M. Hendriks, A. Kastelijn, T. de Hoog, P. van der Walle, and A. Sternbro, "Optical card with parallel readout: Proposal for a new robust storage solution," Proc. SPIE 5380, 10-14 (2004).

[5] J. R. Leger, and G. J. Swanson, "Efficient array illuminator using binary-optics phase plates at fractional-Talbot planes," Opt. Lett. 15, 288-290 (1990).

[6] V. Arrizon, and J. Ojeda-Castaneda, "Talbot array illuminators with binary phase gratings," Opt. Lett. 18, 1-3 (1993).

[7] T. J. Suleski, "Generation of Lohmann images from binary-phase Talbot array illuminators," Appl. Optics 36, 4686-4691 (1997).

[8] P. Xi, C. Zhou, E. Dai, and L. Liu, "Ceneration of near-field hexagonal array illumination with a phase grating," Opt. Lett. 27, 228-230 (2002).

[9] C. Siegel, F. Loewenthal, J. E. Balmer, and H. P. Weber, "Talbot array illuminator for single-shot measurements of laser-induced-damage thresholds of thin-film coatings," Appl. Optics 39, 1493-1499 (2000).

[10] K.-H. Brenner, and R. Buschlinger, "Parallel image scanning with binary phase gratings," J. Europ. 0pt. Soc. Rap. Public. 6, 11024 (2011).

[11] L. P. Bakker, S. Stallinga, and C. Busch, "Binary phase structure for the generation of a periodic light signal," WIPO Patent 2006/035393 (2006).
[12] J. H. Wayland, "A Contrast and resolution improvement in scanning microscopy - viewing sequential signals of light from scanned object, with sequential responses recorded for subsequent reproduction," United States Patent 4806004 (1989).

[13] M. C. Krantz, "Optical system for scanning optical microscopes, has array detector to collect light from object in parallel detection channels for each light spot and construct image of particular field of object," United States Patent 6248988/B1 (1998).

[14] H. Kusunose, "Optical scanner for defect inspection system, moves sample stage relative to light spot matrix array formed by objective lens so as to scan sample surface using two-dimensional spot array," United States Patent 2002/0162979/A (2002).

[15] G. Almogy, and 0. Reches, "Spot grid array imager for automated semiconductor wafer defect inspection, has laser for simultaneously radiating array of spots spaced apart from each other, on surface of semiconductor wafer," United States Patent 2003/0085335/A1 (2003).

[16] D. Gräfe, M. Kühner, and F. Eismann, "Confocal laser scanning microscope has telescope lens system, followed by array of lenslets and lenses with a dichroitic beam splitter, followed by scanning mirrors and focusing lens system," WIPO Patent 2005/033767/A1 (2005).

[17] L. P. Bakker, and R. F. M. Hendriks, "Method and system for scanning an information carrier via one-dimensional scanning," WIPO Patent 2007/043013/A2 (2007).

[18] A. Barty, K. A. Nugent, D. Paganin, and A. Roberts, "Quantitative optical phase microscopy," Opt. Lett. 23, 817-819 (1998).

[19] D. L. J. Vossen, L. P. Bakker, B. Hulsken, and S. Stallinga, “A method of imaging a sample", WIPO Patent 2009/022289 (2009). 